\title{
OPTIMIZATION APPROACH TO THE GUIDANCE AND CONTROL OF MARINE VEHICLES
}

\author{
EVGENY I. VEREMEY \& MARGARITA SOTNIKOVA \\ Department of Computer Systems and Applications, Saint Petersburg University, Russia
}

\begin{abstract}
Modern optimization ideology can be treated now as one of the most powerful instruments for the modeling, analysis and synthesis of various type dynamic systems, including sophisticated automatic controllers for providing guidance and control of marine crafts. Such a situation is explained by universality, flexibility, and convenience of modern optimization methods with respect to relevant marine control applications. This work represents some new results in the development and utilization of the optimization approach for the analysis and design of marine systems such as automatic weather routing, automatic steering, dynamic positioning and others. It is well known that these problems should be considered when taking into account a vast set of requirements, restrictions, and conditions to be satisfied by an appropriate choice of control decision. This determines a specific proposed way to formalize a practical problem in optimization manner. Correspondent partial situations are discussed with orientation to fuel economy, improved reliability and performance enhancement.
\end{abstract}

Keywords: optimization, control system, performance, stability, routing, marine vehicle.

\section{INTRODUCTION}

Due to the intensive development of world economy, there constantly appear many problems connected with a safety of ships motion in a range of increasing marine traffic. These problems seem to be essentially significant for further development of marine transportation services with considerable increasing of their efficiency and reliability.

One of the commonly used approaches to the safety and efficiency ensuring is wide implementation of various automated system for guidance and control application [1], [2], including using such popular systems as marine autopilots, dynamic positioning systems, speed control systems, automatic routing installations, etc.

To guarantee a safe and efficient sailing, the highest quality of automatic control systems operation is in urgent demand. However, as it is well known, a ship motion is extensively influenced by different environmental disturbances such as sea waves, winds, sea currents, change of depth under keel, etc [1]. In view of this remark and many other reasons, it is necessary to state that the operation of various marine automated systems is realized under extremely complicated conditions. This requires taking into account a lot of limitations to be satisfied by the design procedures for these systems.

One of the most popular requirements for the system to be designed is the optimality property. Various analytical methods are known now for the determination of optimal control policies for linear and nonlinear systems subject to a given performance indices [3]. Nevertheless, the optimality is not the end in itself for the most part in practice, as a rule. Rather, the optimization approach should be treated as an effective and convenient instrument to achieve desirable features of the system to be designed. In our opinion, this can be explained by universality, flexibility, and convenience of modern optimization methods with respect to relevant practical implementations of control theory.

This work represents a certain survey of optimization approach applications for marine automated systems design and some new results obtained in this direction. 


\section{GENERAL BACKGROUND FOR OPTIMIZATION APPROACH}

First, remark that the modern complicated practical problems of anyone technical system design are considered taking into account a vast population of requirements, restrictions, and conditions to be satisfied by an appropriate choice of the design result. This obvious statement can be fully referred to various automated systems of marine transport. To provide desirable features of these systems, it is necessary to propose relevant design methods, which guaranty satisfying the mentioned population.

An obvious way to formalize this practical problem with the help of optimization theory can be proposed in the following manner. Defining a desirable set in certain metric space of varying elements on the base of the mentioned population, we can introduce a functional to be minimized as a distance from desirable set. If the numerical solution of this problem allows reaching zero distance, we obtain a correspondent control law with desirable features.

Naturally, the mentioned idea must be defined concretely for every particular situation to provide applicability and effectiveness of implementation. There are a lot of practical cases, which optimization approaches were adopted for; the classical example of such an adaptation is the well-known Response Optimization Tool in MATLAB package.

Let us especially underline that the essence of the initial formulation of the design problem can not be directly connected with a search of extreme values for some functionals, which determine certain performance indices for the systems to be designed. Nevertheless, the optimization ideology can be used overall as a universal approach to attract modern computer technologies for design processes.

To explain the essence of this assertion in details, let us consider some set $X$ of elements $x$ with arbitrary nature, which can be treated as the set of feasible decisions of design problem. Let this collection of elements be a metric space with the metric $\rho\left(x_{1}, x_{2}\right)$, which determines a distance between any two elements $x_{1}, x_{2} \in X$ (Fig. 1).

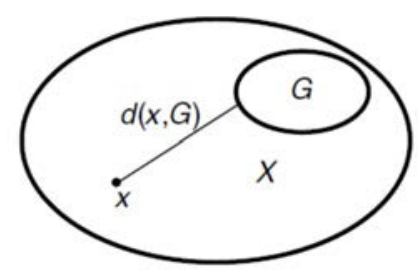

Figure 1: The sets of feasible and admissible decisions of the control design problem.

Let us also assign the subset $G$ (Fig. 1) of admissible decisions within the bounds of the feasible set $X$ : this subset is determined by the whole population of requirements, restrictions, and conditions, which should be satisfied by a design procedure implementation. In essence, any design problem consists in the choice of whatever item $x$ from the given admissible set $G$ (we suppose that it is not empty), and accept this item as a decision of this problem. It is very significant to mark that the question of the mentioned choice in general case has no trivial solution. This can be interpreted as follows: let us take any feasible item $x \in X$. With no special preventive measures, this item does not belong to the admissible set $G$. By other words, introducing the distance from $x$ to $G$ in the form of:

$$
d(x, G)=\inf _{g \in G} \rho(x, g),
$$


obtain $d(x, G)>0$ that is not acceptable.

It is easy to see that the only situation with $d(x, G)=0$ is admissible, which corresponds to a solution of the initial problem. As far as $d(x, G) \geq 0 \forall x \in X$, it is quite suitable to consider the obvious optimization problem:

$$
J(x)=d(x, G) \rightarrow \min _{x \in X}
$$

to minimize a distance (2.1) from any point $x$ to the admissible set $G$. If analytical or numerical solutions of this problem allow reaching zero distance, we obtain a correspondent control law with desirable features.

Especially remark that a majority of numerical methods for the problem (2.2) solution is based on the utilization of necessary conditions of extreme. The most realistic application of these conditions leads to the construction of the items sequence $\left\{x_{i}\right\} \in X$ such that $\mathrm{e}$ relationship $\lim _{i \rightarrow \infty}\left\{x_{i}\right\}=x_{0} \in G$ holds.

Let $X$ be a linear norm space with the introduced norm $\|x\|$, and let the metric is defined as $\rho\left(x_{1}, x_{2}\right)=\left\|x_{1}-x_{2}\right\|$. Given a functional $J(x)$, which is a Frechet differentiable, let suppose that $x_{0} \in X$ is a point of its local extreme. Since the increment of $J$ can be presented as $\Delta J=J\left(x_{0}+h\right)-J\left(x_{0}\right)=d J\left(x_{0}, h\right)+r\left(x_{0}, h\right) \quad \forall h \in X \quad$ with Frechet differential $d J\left(x_{0}, h\right)$ and linear continuous functional $r\left(x_{0}, h\right)\left(\lim r\left(x_{0}, h\right) /\|h\|=0\right.$ for $\|h\| \rightarrow 0)$, then we have the necessary criterion of optimality:

$$
d J\left(x_{0}, h\right)=0 \quad \forall h \in X .
$$

The equality (2.3) can be used as a basis for the mentioned sequence $\left\{x_{i}\right\} \in X$ construction to provide numerical decision of the problem (2.2). For every particular case, the necessary condition (2.3) obtains a concrete form that allows to construct correspondent specialized optimization methods, which are most effective for the concrete situation.

It seems to be quite suitable to remark that for the problem (2.2) it is very easy to check sufficient conditions of extremum for the point $x_{0}$. Really, theirs checking is equivalent to checking of the belonging $x_{0} \in G$.

\section{WEATHER ROUTING SYSTEMS}

The purpose of the weather routing system (WRS) is to assist the ship drivers in deciding, which ship route should be selected in order to perform the optimal voyage. Selection of the ship route is based on two optimization criteria, which must be specified by the user: general time of the motion or general fuel consumption.

Currently, a number of papers are devoted to the problem of WRS-systems design. In particular, the isochrones method was proposed in Zhang and Huang [4]. Another approach is related with a calculus of variations [5] and with dynamic programming [6]. The significant drawback of these approaches is their huge computational complexity. This demands the simplification of the initial problem for possibility of its practical implementation in real-time.

Actually, correspondent optimization problems are traditional subjects for consideration in the range of calculus of variations theory. In general, any variation problem consists of searching out an optimal function $u(x)$, which can be graphically presented as a 
correspondent curve. This function should belong to a certain set $U$ of admissible decisions. As a measure of optimality for a curve, certain real valued functional $J(u)$ should be given, which depends on the function $u(x)$. The simplest example of variation approach in the framework of WRS is to find the shortest curve on a spherical surface of the Earth, connected two given points A and B. This is so called problem of geodesics, which has well known solution: the shortest way is the arc of Great Circle between the mentioned points.

Nevertheless, if we have a set of constraints, limiting the way choice, a solution of the shortest curve problem is not so simple. Naturally, the problem of the ship route optimization becomes still more complicated for the functionals, which determine time of the motion and general fuel consumption for the routes on the surface of the Earth.

A problem of optimal route computation is very important for long-term voyages, for example, for transatlantic transitions. In this case, traveling time is about several days and fuel consumptions reach thousands of tons. These performance indices for a certain ship and selected route are influenced by weather conditions in the sailing zone. Therefore, the problem of optimal weather routing has an essential practical significance.

A solution of weather routing problem usually is complicated by constraints, which must be taken into account. These constraints can be static and dynamic. Coastal lines, shallow waters, zones, restricted for sailing, determine static constraints. These constraints are known in advance for a sailing region. The dynamic constraints are represented by weather alarm zones, for instance by zones with a strong wind, strong currents or high waves height. These zones can be obtained from weather forecast and they are given with a certain probability. The parameters of alarm zones can be specified by the navigator.

The properties of the particular route depend on the parameters of a concrete ship. The same route can be economical for a certain ship and very unprofitable for another one. This is because the weather conditions, for example wind, have a different impact for different ships. Therefore, the parameters of a particular ship should be taken into account for the optimal route design.

In practice, a ship route is often selected by navigator on the base of its own experience. As opposed to this natural approach, consideration of a correspondent optimization problem is proposed with effective algorithms for its approximate solution. These algorithms are based on the finite dimensional approximation of the mentioned variation problems that essentially raises the effectiveness of calculations.

To transform a considered problem to the finite dimensional optimization it is necessary to introduce an aggregate of independent tunable variables, which fully determine the ship route. It can be done in correspondence with the simplified scheme of the motion, where ship trajectory is presented as piecewise azimuth-constant curve (route curve) on the spherical surface of the Earth. The route curve can be presented by the vector:

$$
\mathbf{r}=\left\{\left(\varphi_{1}, S_{1}\right),\left(\varphi_{2}, S_{2}\right), \ldots,\left(\varphi_{p}, S_{p}\right)\right\} \in E^{2 p}
$$

of route variables, where the pair $\left(\varphi_{i}, S_{i}\right)$ represents the $i-t h$ lag: $\varphi_{i}$ is the azimuth and $S_{i}$ is the length of the leg, $p \geq 3$.

Dynamical behavior of the ship on the route curve depends on the frequency of the traction screw rotation. In one's turn, this frequency is fully determined by the given value of desirable ship's speed in a still water. Let introduce the vector $\mathbf{v}=\left(V_{1}, V_{2}, \ldots, V_{p}\right)$ of speed variables. Components of this vector represent desirable constant ship speed for every leg of the route curve. 
Taking into account introduced tunable variables, it is possible to determine ship route trajectory as an aggregate $\gamma_{c}$ of points $\left(\varphi_{g}, \lambda\right)$ belonging to the mentioned piecewise azimuth-constant curve. This aggregate can be presented as follows:

$$
\gamma_{c}=\gamma_{c}(\mathbf{r})=\left\{\left(\varphi_{g}, \lambda\right) \in E^{2}: \lambda \in\left[\lambda_{\min }, \lambda_{\max }\right], \varphi_{g}=\varphi_{g}(\lambda, \mathbf{r})\right\},
$$

where $\lambda_{\min }, \lambda_{\max }-$ minimal and maximal longitude of any spherical rectangular including initial and final points $\mathbf{A}$ and $\mathbf{B}$ of the route. Here function $\varphi_{g}(\lambda, \mathbf{r})$ is defined by well known formulas from the spherical geometry. It is necessary to remark that trajectory depends only on vector $\mathbf{r}$ and is independent on desirable or actual ship's speed.

Obviously, the choice of tunable variables should be done in full correspondence with the weather conditions along the route trajectory. These conditions can be presented by a weather forecast, which represents their evolutions in time. Environmental actions to the ship are realized in accordance with its dynamical features, which determine some constraints for the choice of tunable variables. This dynamic is mathematically represented by differential equation for the ship's speed in the form:

$$
d W / d t=f_{v}\left(t, W, n(V), P_{\text {curr }}, P_{\text {wave }}, P_{\text {wind }}\right) .
$$

Here $W$ is the actual ship's speed, $n(V)$ is the frequency of screw rotation, which depends on desirable speed in a still water; the functions $P_{\text {curr }}=P_{\text {curr }}\left(t, \lambda, \varphi_{g}\right), P_{\text {wave }}=P_{\text {wave }}\left(t, \lambda, \varphi_{g}\right)$ and $P_{\text {wind }}=P_{\text {wind }}\left(t, \lambda, \varphi_{g}\right)$ represent correspondently current, sea waves and wind forces, acting to the ship. All these forces are given by the weather forecast and, in relation to the route trajectory, are the functions of $\lambda, \mathbf{r}$ and $\mathbf{v}$.

Naturally, the mentioned tunable variables are bounded by the correspondent constraints: first, they are determined by the presence of the static forbidden regions for sailing on the spherical surface of the Earth, and second, by the presence of the dynamic forbidden areas, which are depends on the weather conditions.

The construction of WRS-system is based on the following optimization problem:

$$
J=J(\mathbf{r}, \mathbf{v}) \rightarrow \min _{\mathbf{r} \in \mathbf{R}, \mathbf{v} \in \mathbf{V}(\mathbf{r})},
$$

where the performance index $J(\mathbf{r}, \mathbf{v})$ can be specified by the user in two variants: as general time of the motion or as general fuel consumption. Admissible sets $\mathbf{R}$ and $\mathbf{V}(\mathbf{r})$ are determined by the mentioned static and dynamic constraints.

New numerical methods of the problem (3.1) solution are proposed, taking into account the possibility of their real-time implementation. Some of these methods are presented in papers by Veremey and Sotnikova [7] and Wang et al. [8] with practical examples of their applications.

\section{CONTROL LAWS WITH MULTIPURPOSE STRUCTURE}

As it was mentioned above, all modern practical approaches, which are used for a design of various automated marine systems of control, take into account a vast population of requirements, restrictions, and conditions to be satisfied by a choice of control laws. Specifically, this is true for such popular systems as marine autopilots and dynamic positioning controllers. Here an advanced optimization ideology is proposed for these systems construction, using specific multipurpose (MP) structure of control laws. This 
structure contains two parts, the first of which is used constantly, and the second can be switched on or off, as needed.

\subsection{Marine autopilots design}

Let remark that various issues associated with the design of autopilots for marine surface vessels have already been extensively researched [1], [2], [9]. However, essentially less attention is paid to the feasibility of autopilots turning during its operation depending on the actual conditions of sailing. Let us consider the autopilot control law with MP-structure to provide its varying elements optimal tuning depending on specific regime of ship's motion.

Standard problems of autopilot design are usually considered with respect to the linear state space model of a ship motion. This model can be obtained using linearization of the initial nonlinear ship's equations in the neighbourhood of its equilibrium position, which corresponds to the given regime of motion with a constant speed. Suppose that the following LTI-system represents the model of a ship as a controlled plant:

$$
\begin{aligned}
& \dot{\mathbf{x}}=\mathbf{A x}+\mathbf{b} \delta+\mathbf{h} d(t), \\
& \mathbf{e}=\left(\begin{array}{ll}
\lambda \cdot \mathbf{c x} & \delta
\end{array}\right)^{\mathrm{T}}, \\
& y=\mathbf{c x} \text {. }
\end{aligned}
$$

Here $\mathbf{x} \in E^{n}$ is the state space vector, scalar $\delta \in E^{1}$ denotes the rudder deflection, $d \in E^{1}$ presents the external disturbance, $y \in E^{1}$ is the yaw angle treated as a measured variable, and $\mathbf{e} \in E^{2}$ is the controlled vector, $\lambda \geq 0$ is the given real number (the weight coefficient). All the matrices $\mathbf{A}, \mathbf{b}, \mathbf{h}$ and $\mathbf{c}$ with constant components have correspondent dimensions. Suppose that the system (4.1) is fully controllable by $\delta$ and observable by $y$.

In addition, let also introduce the following linear model of an actuator:

$$
\dot{\delta}=u
$$

where $u \in E^{1}$ is the control signal.

Let's accept the following structure of control law (controller) to stabilize marine ship motion as it was justified in Veremey [10], [11]:

$$
\begin{aligned}
\dot{\mathbf{z}} & =\mathbf{A z}+\mathbf{b} \delta+\mathbf{g}(y-\mathbf{c z}), \\
\xi & =F(s)(y-\mathbf{c z}), \\
u & =\widetilde{\mathbf{k} z}+k_{0} \delta+\widetilde{v} y+\xi .
\end{aligned}
$$

It is easy to see that the introduced structure consists of three items: the first equation presents an asymptotic observer, the second one describes so called "dynamical corrector" and the third equation composes a control signal for rudder's actuator. Here $\mathbf{z} \in E^{n}$ is the state space vector of asymptotic observer, $\mathbf{g}$ is the given column matrix such that the matrix A- ge is Hurwitz.

To tune an autopilot control law with the MP-structure (4.3), it is necessary to search three initially unknown elements: vector $\mathbf{g}$ for the observer, transfer function $F(s)$ for the corrector, and parameters $\tilde{\mathbf{k}}, \mathrm{k}_{0}$ and $\tilde{v}$ for the composer.

As it is shown in Veremey [10]-[12], a general design procedure for the MP-controller tuning can be decomposed into consequent numerical solutions of the three following 
synthesis problems. The first of them provides a choice of the parameters $\mathbf{k}$ and $k_{0}$ for the basic stabilizing controller $\mathrm{u}=\mathbf{k} \mathbf{x}+\mathrm{k}_{0} \delta$ with the plant (4.1), (4.2), where $\mathbf{k}=\tilde{\mathbf{k}}+\tilde{\mathrm{v}} \mathbf{c}$. This step optimizes an own motion of the closed-loop connection with no disturbances action. The second step is directed to the search of the vector $\mathbf{g}$. It is appropriate to note that the controller (4.3) provides an integral action with respect to input $d$ and output $y$ for the closed-loop system, if additional requirement for the function $F(s)$ holds. Taking into account this action, a choice of the vector $\mathbf{g}$ is oriented to the optimization of the motion under actions of bias disturbances $d(t)$ with low frequencies.

At last, the third synthesis problem is devoted to the search of the transfer function $F(s)$ of the corrector to provide desirable dynamics of the closed-loop system under actions of disturbances $d(t)$ with relatively high frequencies (sea waves). As it is shown in Veremey [10]-[12], for this case we need to minimize the functionals, which are presented by the norms of the matrix $\mathbf{H}_{d \mathbf{e}}$ from $d$ to $\mathbf{e}$ for (4.1)-(4.3) in the sense of the spaces $H_{2}$ or $H_{\infty}[13]$.

As a result, one can conclude that if all the mentioned problems are successfully solved, we fully determine the feedback (4.3), which guaranties desirable features of the closed-loop connection for all regimes of ship's motion.

Let us especially underline that the main goal of MP-structure using is to expand the idea of a separate optimal tuning for all units of control law. In our opinion, this idea, which is not fully realized in the range of existing approaches, provides essential flexibility of the control law. Such flexibility is granted by the structure (4.3), where the corrective term can be selected subject to a current regime of the ship motion.

\subsection{Dynamic positioning systems}

The problem of dynamical positioning (DP) vessel control is very significant in the area of marine control systems analysis and design. Now DP systems are widely used for the various types of marine vessels in different marine connected branches such as hydrography, inspection of marine construction, wreck investigation, underwater cable laying, and so on. The exhaustive survey of DP control systems is done in Sørensen [14].

Similar to marine autopilots, existing approaches to DP systems design have some disadvantages, which can be overcame in the mentioned above manner, using multipurpose structure of control laws [15], [16].

Actually, let us consider nonlinear mathematical model of DP vessel as

$$
\begin{aligned}
& \mathbf{M} \dot{\mathbf{v}}=-\mathbf{D} \mathbf{v}+\boldsymbol{\tau}+\mathbf{d}(t), \\
& \dot{\boldsymbol{\eta}}=\mathbf{R}(\boldsymbol{\eta}) \mathbf{v} .
\end{aligned}
$$

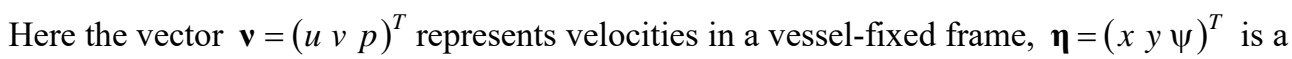
joint position $(x, y)$ and a heading angle $\psi$ vector relative to an Earth-fixed frame. Vector $\boldsymbol{\tau} \in E^{3}$ implies a control action generated by the propulsion system, vector $\mathbf{d} \in R^{3}$ is provided by the external disturbances of any nature. Matrices $\mathbf{M}$ and $\mathbf{D}$ with the constant elements are positive definite, $\mathbf{M}=\mathbf{M}^{T}, \mathbf{R}(\boldsymbol{\eta})=\mathbf{R}(\psi)$ is rotation matrix.

By analogy with (4.3), let us introduce nonlinear control law: 


$$
\begin{aligned}
& \mathbf{M} \dot{\mathbf{z}}_{v}=-\mathbf{D} \mathbf{z}_{v}+\boldsymbol{\tau}+\mathbf{R}^{T}(\boldsymbol{\eta}) \mathbf{K}_{1}\left(\boldsymbol{\eta}-\mathbf{z}_{\eta}\right), \\
& \dot{\mathbf{z}}_{\eta}=\mathbf{R}(\boldsymbol{\eta}) \mathbf{z}_{v}+\mathbf{K}_{2}\left(\boldsymbol{\eta}-\mathbf{z}_{\eta}\right), \\
& \xi=\mathbf{F}(s)\left(\boldsymbol{\eta}-\mathbf{z}_{\eta}\right), \\
& \boldsymbol{\tau}=-\mathbf{K}_{d} \mathbf{z}_{v}-\mathbf{R}^{T}(\boldsymbol{\eta}) \mathbf{K}_{p}\left(\mathbf{z}_{\eta}-\boldsymbol{\eta}_{d}\right)+\xi .
\end{aligned}
$$

Note that a control law of the form (4.5) has MP-structure with the same tuneable items as for (4.3). A general design procedure for theirs tuning also can be decomposed into consequent numerical solutions of the three mentioned above synthesis problems, which can be realized, using optimization approach. Some details and examples of this approach implementation are presented in Sotnikova and Veremey [15] and Veremey [16].

\section{TRACKING CONTROL AND OPTIMAL DAMPING}

Let us consider nonlinear mathematical model for controlled marine vehicle of the form:

$$
\dot{\overline{\mathbf{x}}}=\mathbf{F}(\overline{\mathbf{x}}, \overline{\mathbf{u}})
$$

where $\overline{\mathbf{x}} \in R^{n}, \overline{\mathbf{u}} \in R^{m}$, and suppose that for any $\overline{\mathbf{u}}(t) \in U$ the conditions for the existence and uniqueness of the initial problem solution hold.

Let a desirable reference motion of the system (5.1) be given as the functions $\mathbf{x}_{\rho}(t), \mathbf{u}_{\rho}(t)$, satisfying the eqn:

$$
\dot{\mathbf{x}}_{\rho}(t) \equiv \mathbf{F}\left(\mathbf{x}_{\rho}(t), \mathbf{u}_{\rho}(t)\right) .
$$

Let us introduce deflections of the real motion from the reference one:

$$
\mathbf{x}:=\overline{\mathbf{x}}-\mathbf{x}_{\rho}, \mathbf{u}:=\overline{\mathbf{u}}-\mathbf{u}_{\rho},
$$

and after substitution $\overline{\mathbf{x}}=\mathbf{x}+\mathbf{x}_{\rho}, \overline{\mathbf{u}}=\mathbf{u}+\mathbf{u}_{\rho}$ into (5.1) obtain:

$$
\begin{gathered}
\dot{\mathbf{x}}+\dot{\mathbf{x}}_{\rho}=\mathbf{F}\left(\mathbf{x}+\mathbf{x}_{\rho}, \mathbf{u}+\mathbf{u}_{\rho}\right) \Leftrightarrow \dot{\mathbf{x}}=\mathbf{G}(t, \mathbf{x}, \mathbf{u}), \\
\mathbf{G}(t, \mathbf{x}, \mathbf{u}):=\mathbf{F}\left(\mathbf{x}+\mathbf{x}_{\rho}(t), \mathbf{u}+\mathbf{u}_{\rho}(t)\right)-\mathbf{F}\left(\mathbf{x}_{\rho}(t), \mathbf{u}_{\rho}(t)\right) .
\end{gathered}
$$

It is obvious that the system (5.4), which is presented in deflections from reference motion, has zero equilibrium $\mathbf{G}(t, \mathbf{0}, \mathbf{0})=0 \quad \forall t \geq 0$.

The problem of tracking control is to determine the control inputs $\overline{\mathbf{u}}$ such that the real motion of the vehicle follows a given reference $\mathbf{x}_{\rho}(t), \mathbf{u}_{\rho}(t)$ as good as possible. In a range of mathematical formalization, this means that the control $\mathbf{u}$ should be designed as a feedback connection:

$$
\mathbf{u}=\mathbf{u}(t, \mathbf{x}),
$$

which stabilizes the origin or provides asymptotic stability of the zero equilibrium for the system (5.4).

If the controller (5.5) is designed, then the tracking control:

$$
\overline{\mathbf{u}}(t, \overline{\mathbf{x}})=\mathbf{u}\left(t, \overline{\mathbf{x}}-\mathbf{x}_{\rho}(t)\right)+\mathbf{u}_{\rho}(t)
$$


guaranties that starting from any initial state in a region $B_{x}$ of stability, the tracking errors (5.3) tend to zero subject to $t \rightarrow \infty$.

It is necessary to remark that the complexity of nonlinear feedback control challenges us to come up with systematic design procedures to meet control performance objectives and synthesis specifications.

The common approach to control design is based on optimization problems:

$$
J=J(\mathbf{u}) \rightarrow \min _{\mathbf{u} \in U}
$$

with the integral functional of the form:

$$
J=J(\mathbf{u}):=\int_{0}^{\infty} \ell(t, \mathbf{x}(t), \mathbf{u}(t)) d t,
$$

which is given on the motions of the controlled plant (5.4), $\ell(\cdot) \geq 0$.

At present time, numerous approaches are well known for a practical solution of the problem (5.7), (5.8). They are based on the Pontryagin's Maximum Principle, on the Bellman's Dynamic Programming Principle (using HJB-equation), on the finite-dimensional approximation in the range of MPC technique, etcetera. Unfortunately, all these approaches are connected with the huge extent of calculations that essentially impedes their implementation in real time regimes of control.

Here different idea is proposed to design stabilizing tracking controllers, which is based on the theory of transient processes optimal damping (OD). This theory, which was firstly proposed and developed by Zubov [17]-[19], gives effective analytical and numerical methods for control calculation with essentially reduced computational consumptions.

In framework of OD-theory, a central role plays initially given Lyapunov function candidate $V=V(t, \mathbf{x})$ and an auxiliary functional:

$$
L=L(t, \mathbf{x}, \mathbf{u})=V(t, \mathbf{x})+\int_{0}^{t} f_{0}(\tau, \mathbf{x}(\tau), \mathbf{u}(\tau)) d \tau
$$

to be damped optimally. Its rate of change along the solutions of the system (5.4) can be presented as follows:

$$
\begin{aligned}
& W=W(t, \mathbf{x}, \mathbf{u}):=\left.\frac{d L}{d t}\right|_{(5.4)}=\left.\frac{d V}{d t}\right|_{(5.4)}+f_{0}(t, \mathbf{x}, \mathbf{u})=\frac{\partial V}{\partial t}(t, \mathbf{x})+ \\
& +\left.\frac{\partial V}{\partial \mathbf{x}}(t, \mathbf{x}) \dot{\mathbf{x}}\right|_{(5.4)}+f_{0}(t, \mathbf{x}, \mathbf{u})=\frac{\partial V}{\partial t}(t, \mathbf{x})+\frac{\partial V}{\partial \mathbf{x}}(t, \mathbf{x}) \mathbf{G}(t, \mathbf{x}, \mathbf{u})+f_{0}(t, \mathbf{x}, \mathbf{u}) .
\end{aligned}
$$

The essence of OD-approach consists of the control generation as a function from the current values of variables $t, \mathbf{x}$ in the form:

$$
\mathbf{u}_{0}=\mathbf{u}_{0}(t, \mathbf{x})=\arg \min _{\mathbf{u} \in U} W(t, \mathbf{x}, \mathbf{u}) .
$$

Under certain conditions to be satisfied by the choice of $V=V(t, \mathbf{x})$, this function becomes Lyapunov function that guaranties an asymptotic stability of the origin for the system (5.4) with the help of the controller (5.11). 
Let us remark separately that for some cases, the control $\mathbf{u}_{0}(t, \mathbf{x})$ can be derived analytically, but for the most situations, this one is calculated numerically.

As an example of these approach applications, a solution of the problem of marine ship's heading and forward speed tracking control design can be presented.

\section{EXPERT CONTROL SYSTEMS}

Let us remark that all optimization problems, which were discussed above, can be realized fully automatically without the using of direct human support. Nevertheless, this is not the only situation for many various applications. This is connected with the design and exploitation of exclusively sophisticated systems, when the strict mathematical statement of optimization problems is essentially impeded.

As for marine automated systems, let us consider the routine situation, when it is necessary to design complicated controllers, including a huge number of various elements. These elements should be integrated into varying hierarchical structure to be tuned in real-time regime of ships motions. It is obvious that there are many difficulties connected with the mathematical representation of such systems at the formal level. In particular, it seems to be very problematical to reflect a performance of this system by any unique functional: moreover, the majority of performance indices here can be determined only informally on the base of the expert's opinions.

The absence of the formal functionals essentially obstructs implementation of the mentioned generalized idea to minimize a distance from any feasible decision to the desirable set of control laws. Nevertheless, there exists a possibility to implement the optimization ideology in this case too, using a special computer-based procedure for a treatment of no formalized expert's preferences. Let us consider the main positions of this ideology, drawing special attention to a distribution of the actions between the expert and computational part of the system.

To begin with, let suppose that a ship's control system to be designed has certain given constant part of its structure, and also includes a varying part $P$ (the aggregation of transfer matrices, numerical parameters, nonlinear blocks and so on) to be chosen as a result of design.

To estimate a performance of a designed control system, the expert should obtain appropriate information about its actual behavior and features. This is a function of a computer environment, supporting actions of the expert, to reconstruct correspondent modeling situation $S(P)$, describing the variant $P$ to be estimated by the expert.

The informal performance estimation is based on the comparison of two any modeling situations $S\left(P_{1}\right)$ and $S\left(P_{1}\right)$ by the expert, who can uniquely choose the best of them. This means that if we have the population of varying parts $P_{1}, P_{2}, \ldots, P_{K}$ with the correspondent modeling situations $S\left(P_{1}\right), S\left(P_{2}\right), \ldots, S\left(P_{K}\right)$, then the expert always can range the mentioned population, for example, as follows:

$$
S\left(P_{1}\right) \succ S\left(P_{2}\right) \succ \ldots \succ S\left(P_{K}\right) \Rightarrow P_{1} \succ P_{2} \succ \ldots \succ P_{K} .
$$

Remark that if a system of the expert's preferences is transitive, then there exists some functional $\Phi(P)$, which is objectively reflected by this system: nevertheless, a formal representation of the mentioned functional is unknown for the expert.

Let restrict our discussion by the simplest case, when the varying elements are adjustable numerical parameters of the control system jointed into the vector $\mathbf{h} \in E^{p}$. As an example of this situation, tunable parameters of the multipurpose structure can be accepted: coefficients of the basis control law or coefficients of the observer presented in Section 4. 
For this case, the objective performance index is the function $\Phi(\mathbf{h})$ of $p$ real variables: let suppose that this function should be minimized. To this end, starting from the initial point $\mathbf{h}^{0}$, computer environment should propose a new point $\mathbf{h}=\mathbf{h}^{*}=\mathbf{h}^{0}+\rho \mathbf{e}$ such that $S\left(\mathbf{h}^{*}\right) \succ S\left(\mathbf{h}^{0}\right)$, where $\mathbf{e} \in E^{p}(\|\mathbf{e}\|=1)$ is a direction of descent, and $\rho$ is the size of the step in this direction. To provide a computation of the mentioned points, it is quite suitable to use the Nelder-Mead Simplex method because of this one does not use formal representation of gradients. Moreover, using the mentioned method we do not need to calculate the values $\Phi(\mathbf{h})$ : here we can directly apply a system of the expert's preferences.

Actually, the Nelder-Mead method is based on the analysis of a simplex in $p$-dimensional space, which is characterized by the $p+1$ distinct vector that represents its vertices. At each step of the descent, a new point in the neighborhood of the current simplex is generated. Next, the expert compares the modeling situation at the new point with the situations for the vertices of the simplex and, usually, one of the vertices is replaced by the new point, giving a new simplex. Then these actions are repeated until the measure of the simplex is less than the specified tolerance.

As a result, we arrive at the simple scheme of the mutual interaction between the expert and computer environment. The role of the expert consists of the assignment of initial point $\mathbf{h}_{0}$ and initial size $\rho$ : then he applies his system of the preferences at each step of the parameters search. Computer environment generates the current simplex and proposes correspondent modeling situations to be estimated by the expert for every vertex of the current simplex.

Actually, the approach proposed above can be expanded for situations that are more general with respect to the varying part $P$ and functionals $\Phi(P)$ for the marine automated system to be designed.

\section{CONCLUSION}

This work is presented to survey and discuss various utilizations of the modern optimization ideology for modeling, analysis and synthesis of automatic marine controllers. Certain attention is paid to the fundamental background of optimization approach application for the design of control system taking into account a vast population of requirements, restrictions, and conditions to be satisfied by an appropriate choice of control laws. All the presented examples illustrate the concrete practical problems connected with automated marine systems design, which can be effectively solved using numerical optimization methods.

Special attention is devoted to the weather routing system: new numerical methods for theirs design are proposed, taking into account the possibility real-time implementation. An advanced optimization method is developed for the synthesis of marine autopilots and dynamic positioning controllers. Note that these methods are substantively based on the using of the specialized MP-structure of control laws to be designed. Significant issues are also discussed with relation to the tracking controller synthesis and to the expert systems utilization with the help of optimization approach.

The results of executed investigations presented above can be expanded to take into account robust features of automatic controllers, and to take into account transport delays both in input and output of controlled plants. 


\section{ACKNOWLEDGEMENT}

This work was supported by the Russian Foundation for Basic Research (RFBR) (Project 1707-00361) controlled by the Government of Russian Federation.

\section{REFERENCES}

[1] Fossen, T.I., Handbook of Marine Craft Hydrodynamics and Motion Control, John Wiley \& Sons: Chichester, 2011.

[2] Perez, T., Ship Motion Control: Course Keeping and Roll Stabilization using Rudder and Fins, Springer-Verlag: London, 2005.

[3] Grimble, M.J., Robust Industrial Control Systems - Optimal Design Approach for Polynomial Systems, John Wiley \& Sons: Chichester, 2006.

[4] Zhang, J. \& Huang, L., Optimal ship weather routing using isochrones method on the basis of weather changes. International Conference on Transportation Engineering, pp. 2650-2655, 2007.

[5] Bijlsma, S.J., A computational method for the solution of optimal control problems in ship routing. Navigation. Journal of the Institute of Navigation, 48, pp. 145-154, 2001.

[6] Wei, S. \& Zhou, P., Development of a 3D dynamic programming method for weather routing. The International Journal on Marine Navigation and Safety of Sea Transportation, 6(1), pp. 79-85, 2012.

[7] Veremey, E.I. \& Sotnikova, M.V., Optimal routing based on weather forecast. International Journal of Open Information Technologies, 4(3), pp. 55-61, 2016. (In Russian.)

[8] Wang, H.B., Li, X.G., Li, P.F., Veremey, E.I. \& Sotnikova, M.V., Application of realcoded genetic algorithm in ship weather routing. Journal of Navigation, 71(4), pp. 989-1010, 2018.

[9] Hammound, S., Ship motion control using multi-controller structure. Journal of Maritime Research, 9(1), pp. 45-52, 2012.

[10] Veremey, E.I., $\mathrm{H}_{\infty}$-Approach to wave disturbance filtering or marine autopilots. IFAC Proceedings Volumes (IFAC PapersOnline), 9(1), pp. 410-415, 2012.

[11] Veremey, E.I., Optimization of filtering correctors for autopilot control laws with special structures. Optimal Control Applications and Methods, 37(2), pp. 323-339, 2016.

[12] Veremey, E.I., Dynamical correction of control laws for marine ships' accurate steering. Journal of Marine Science and Application, 13(2), pp. 127-133, 2014.

[13] Veremey, E.I., Special spectral approach to solutions of SISO LTI H-optimization problems. International Journal of Automation and Computing, 16(1), pp. 112-128, 2019.

[14] Sørensen, A.J., A survey of dynamic positioning control systems. Annual Reviews in Control, 35(1), pp. 23-136, 2011.

[15] Sotnikova, M.V. \& Veremey, E.I., Dynamic positioning based on nonlinear MPC. IFAC Proceedings Volumes (IFAC PapersOnline), 9(1), pp. 31-36, 2013.

[16] Veremey, E.I., Separate filtering correction of observer-based marine positioning control laws. International Journal of Control, 90(8), pp. 1561-1575, 2017.

[17] Zubov, V.I., Oscillations in Nonlinear and Controlled Systems, Sudpromgiz: Leningrad, 1962. (In Russian.)

[18] Zubov, V.I., Theory of Optimal Control of Ships and Other Moving Objects, Sudpromgiz: Leningrad, 1966. (In Russian.)

[19] Zubov, V.I., Theorie de la Commande, Mir: Moscow, 1978. 